\title{
Fuzzy Knowledge Base of Operational Command and Control Information System
}

\author{
Rui Guo ${ }^{1,}$, Xinye Zhao ${ }^{1, b}$ and Chengzhi Gao ${ }^{1, c}$ \\ ${ }^{1}$ Dalian Naval Academy, Dalian 116018, China \\ amsegr@163.com, b'zhaoxinye@nudt.edu.cn, cgaochengzhi1981@sina.com
}

\begin{abstract}
Keywords: Operational Command and Control Information System; Fuzzy Knowledge Base; Fuzzy Reasoning; Battle Simulated Entity.

Abstract.

Decision of commanders requires a great deal military knowledge and situation information. For operational command and control information system, the construction of control and command model requires more detailed knowledge, and the completion of the knowledge base can dispose of the responsibility to the running speed and efficiency of control and command model. However, decisions in real battlefield consisting of too much fuzzy information, so it is acknowledged that how to deal with fuzzy information enables a significant portion of the development of knowledge base. After delving into knowledge base of military analysis and simulation evaluation system, the design of fuzzy extended data model is presented. Moreover, its correctness by an illustrative example has been demonstrated.
\end{abstract}

\section{Introduction}

With the advent of the era of information warfare, the form and mode of operation of the war has undergone tremendous changes. For the implementation of large-scale joint operations and diversified military mission planning needs, operational command and control information system (OCCIS) should be equipped with multiple combat forces, multidimensional battlefield space, a variety of action patterns, etc. All kinds of security systems based on the integration of information systems system simulation should have the capabilities to support the joint combat program demonstration and optimization, combat plan auxiliary test and deduction evaluation, as well as ombat implementation process of aircraft auxiliary decision-making, joint combat exercises, and combat power development demonstration and other functions, by accelerating the entire operational command and control of the information chain to comprehensively enhance the combat effectiveness of the army[1,2].

The establishment of command and control (C2) model requires detailed knowledge support, and the completeness of knowledge base (KB) has a decisive influence on the speed and efficiency of command and control model [3]. Most of the models with command and control capability in foreign countries are equipped with corresponding knowledge base. $\mathrm{KB}$ is classified into various operational regulations, tactical principles, rules of warfare and classic warfare, etc., for the command and control model call, reliable decision planning.

But in the commander's decision-making, the reasoning process is often similar. For example, according to the conditional statement (hypothetical) "if the weather is good", the "strategic deployment conditions are mature" and the premise (bluntly) "If the weather is very good", immediately concluded that "strategic deployment conditions are very mature." This inaccurate reasoning cannot be done with classical binary logic or multi-valued logic.

And fuzzy reasoning can be better applied to such cases [4]. Fuzzy reasoning is based on fuzzy set theory, which is a kind of uncertainty reasoning, which is based on general set theory. It is of great significance in the development of artificial intelligence technology. The commander model can use the traditional production rules and other techniques, combined with fuzzy reasoning function, to complete the situation assessment, combat programs and action options. And the staff support part is able to complete the mobile plan, then the supply plan, fire support coordination. Fuzzy knowledge 
base includes the storage of enemy and friendly command and decision information for combat operations to select and build commander behavior model.

\section{Modeling of Command and Control Model in OCCIS}

In OCCIS, all simulators are consistent and equal. Through the modeling of "Observe-Orient-Decide-Action (OODA) [5], both sides can judge by the situation. On their own most favorable combat program. To this end, OCCIS extensive use of artificial intelligence methods commander modeling: The user can enter the two warring sides Force troops plan; the establishment of operational mechanisms for decision-making, provide rules processor; man-machine interface that allows users to change Command control model to determine the value of key variables; provide behavior templates and planning strategies, resulting in a certain period of time need to simulate the implementation of the tasks and plans. At the same time, OCCIS provides a rich commander model to complete the Situation Assessment and Action Options (COA, Course of Action) selection, to provide commander behavior model (CBM, Commander Behavior Model), to achieve a variety of programs to determine and decision The Commander behavior model using hybrid artificial intelligence system, by way of fuzzy rule sets of rules and regulations to combat modeling and COA were selected so scalable performance for trend analysis, and the use of the board of competition policy enforcement judgment.

OCCIS system design, and implementation of the abstract entity for all levels of decision-making thought process real combat first aid command and control components, the decision-making process and decision-making capabilities separated from the simulation model; then depends on the law of military simulation entities activity or course of action model Data, military rules and behavioral characteristics of abstract modeling, the use of a series of "If-Then-Else" form of heuristic rules to express knowledge. The core idea of the design is to build the combat simulation entity into a model engine whose decision-making process and behavior process are abstracted into content that can be viewed, understood, modified and configured from the outside.

In military combat simulation, command and control system with real-time, dynamic uncertainties and mass characteristics by constructing multi-Agent architecture to describe command and decision-making processes, the use of complex interactions between multiple command decisions effectively achieve operational Agent Simulation of the application of the target. Each combat simulation entity in OCCIS has a certain command and control capability (self-decision making ability). The complexity of the entity's function determines that different entities have different command and control capabilities. For example, a land-mine-field command and control entity to perform a basic function, namely to prevent land entities try to break out of the minefield; an infinite super-complicated decision-making process is Air Force Commander issued a joint force of the air tasking order. By adding a command control to the associated entity, a large number of entities will have a certain command and control function.

\section{FKB of OCCIS}

FKB (Fuzzy Knowledge Base) of OCCIS is the realization of the expert system, which exposes the decision logic to the user in the form of data. The knowledge base in OCCIS is implemented as a plug-in, and the knowledge base plug-in can be assigned to any entity that participates in the battle, or whose command control component has a knowledge base plug-in interface. At least one knowledge base plug-in is required for any command and control component that wants to set up its joint operational command entity, the joint combat forces, and the joint level of intelligence. The knowledge base plug-in must be able to reason itself, enemies or environmental information, and the reasoning process must be made acceptable to the user, through the knowledge base to support the command and control model. 


\section{Composition of FKB}

FKB consists of three basic elements: Facts, Rules, and Actions. Facts indicate the information needed for decision-making; rules are used to correlate facts and to compute their interrelations; action is a mechanism for responding to the outside world based on rules.

\section{Facts}

The information inferred in the knowledge base is expressed by a series of facts. The fact can be achieved by numerical data (eg, quantity, percentage, time, etc.), boolean type data (eg, on position/absent, on plan/out of plan, etc.) or text information (eg, clear alerts, etc.) to describe. Facts are used to describe different types of information.

In FKB, an entity needs to consider the information is called the fact that it is the rule of the drive. The knowledge base interacts with the simulation through a series of facts. Based on the factual value of the fact, it is judged whether the user-defined action can be triggered. For example, when a combat entity receives an "UnderFire" message, the phase is changed from the "REHOSTILITIES" state to the "HOSTILITIES" state, and a set of commands will be triggered so that the corresponding Entities battle with enemy entities, and their weapons systems will become "free" (war free) "state.

\section{Rules}

Facts establish rules by evaluating the values associated with them. One or more rules can be used to create fact values. Rules are always evaluated in the order specified by the user interface until the conditions of the rule are met. Once the conditions of the rule are met, the fact will set the corresponding value.

The fact that a single "If-Then-Else" rule will render two values. If a fact needs to have more than two values, such as a stage or state, or if it has a complex set of conditions, more rules will increase. Having multiple rules Facts should be formatted into multiple "If-Then" rules and a final statement. The final declaration provides the user with a choice of selecting an "If-Then-Else" rule, or a static value (such as Ture or Default), or using the value of the factual conversion itself as a reference. Once the "If-Then-Else" rule is used, it is generally put in the end, "Else" covers all the rest of the conditions, and the logical calculation. The following fragment code 1 is used to determine the true and false status of the declaration after the "moveOrder" rule is employed, in which an excerpt of the ontology is shown in Fig.1. If the issue of the mobile command is issued within the range of [200,300], or if the move command is complete, or "the final activity of the mobile command is attack" False, the statement is false, otherwise true.

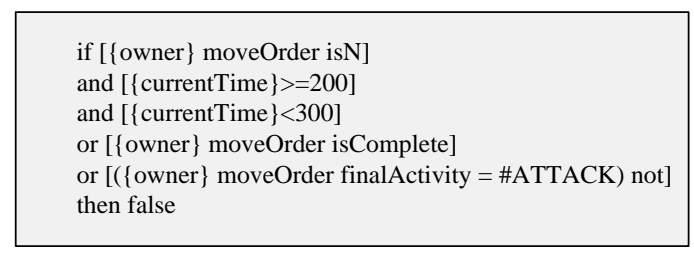

Fig.1 segment code of “moveOrder” Rule

\section{Actions}

Knowledge Base behavior is responsible for setting fact values, sending commands, and stimulating related behavior in simulation deductions. Different knowledge bases have different behaviors for different decisions. All knowledge bases have the ability to set fact values.

The execution of the act depends on the change of the factual assessment - the fact that it is estimated to be a different value from its previous value (for example, stage from stage 1 to stage 2). After the value is changed, the new value is searched for in the list of values for the specified value. If necessary, the behavior can be defined as every possible fact value. The fact that the input and control types do not allow the execution of the behavior, because they only allow a value at the beginning of the simulation and do not know the value after that. 
Behavior is performed only when the value of the fact is changed, so that the behavior is not executed in the course of the factual assessment. However, if a fact value returns another value, and there is no declaration before, the behavior will be executed the next time.

\section{Application of FKB}

\section{Application Pattern of FKB}

FKB uses a plug-and-play mode for any entity unit. According to the customized data of different combat situations, different facts, rules and actions are selected according to different combat entity units in the existing fuzzy knowledge base, and the global instances of the fuzzy knowledge base belongs to them, and they are allocated. To each entity unit or entity group. Each individual unit fuzzy knowledge base will be the result of the corresponding facts, rules and actions, as shown in Figure 2. The system provides the user with a graphical user interface (GUI) for intuitive building of a FKB instance.

For OCCIS, where high-level facts, rules and associated behaviors are not fully applicable to all tactical firepower. For high-level entity units, emphasis is placed on the development of the facts, rules and actions associated with tactical and tactical, technical, and process development rather than theater-level decisions.

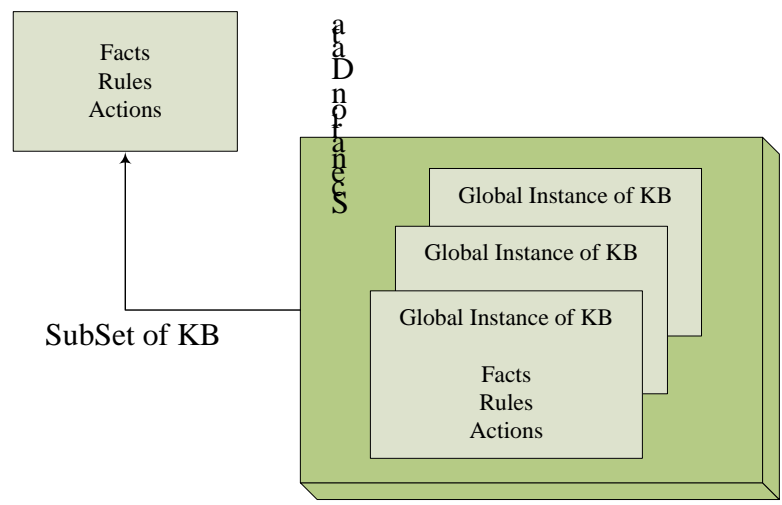

Fig.2 The application pattern of FKB

\section{Reasoning Application of FKB}

The following is an example of the threat assessment in the navy data fusion, and introduces the application of the fuzzy knowledge base in the naval command and control model. The algorithm comprehensively evaluates the enemy's proximity to our forces and our military positions, and points out the threat level [6]. The joint force command and control model makes quick decision-making based on the results.

The data fusion algorithm in OCCIS involves reasoning on unrecognized resources. But the integration of unrecognized resources is computationally large and unnecessary. It is meaningful to analyze resources only when certain types of operational simulation entities can be uniquely identified by certain special resources. Thus, the fuzzy knowledge base adds a reasoning layer. First, the user must define the combat simulation entity to make the fuzzy reasoning of the fact "AgentTypesForResourceAnalysis"; then, call the existing fact "SubmarineType" to analyze and return the entity type. When a resource is not analyzed for an entity, the entity is always parsed as the first level of the entity type. Entities that cannot be blurred are to map their resources to real resources, while entities that can blur the fusion build their knowledge of the resources and gradually acquire the names of real resources from unknown resources.

The algorithm includes two key factors: combat capability and proximity time. According to the quantity and type of the weapon model, we use the standard unit of armament (SUA) parameter of the weapon and equipment to evaluate the combat capability score of each threat unit, and obtain the first factor of the algorithm. The two factors are related to the location of the threat unit, the proximity of 
the speed and the range of our troops. Generally speaking, the closer the threat unit is to a given location, the greater the threat is, and the threat at different distances is not the same Type missile missiles are more threatening than torpedoes because they move fast; the threat of bullets is greater than the threat of ordinary missiles because they use extended range weapons models.

Allows the user to set a relative threat value for each type of ship protection threat system (by defining a "maximum number of threats" - the system generates the maximum threat value of 100 for a region), and based on this value, Interpolate a value between 0 and 100. Find all the ground-to-air systems that can assess the threat of the target, and use the fuzzy logic system to handle the threat values given by each ground-to-air system, resulting in an overall target area threat (0 to 100). The fuzzy logic uses the formula: $A+B-A^{*} B$, where $A$ and $B$ are the threat values given by each ground-to-air system, normalized to between 0 and 100 . The user can also define the "risk tolerance" of the aircraft platform's calculated threat value, which is done by defining a range, and then the air defense plan matches the risk tolerance of the ship's platform with the estimated threat value.

\section{Conclusion}

This paper is directed to OCCIS. By expanding the fuzzy knowledge base plug-in function of the command and control model, the command and control model can exchange the information of the battlefield through the perception function and the battlefield environment as the prerequisite for the judgment and reasoning of the command and control model. Compared with the source trend in the knowledge base, real-time fuzzy reasoning is carried out, and the function of planning and decision-making of command and control model is completed, which greatly enhances the artificial intelligence level and decision-making credibility of the simulation and control system command and control model.

\section{Acknowledgements}

This work is supported by China Postdoctoral Science Foundation under Grant No.2016M602962 and No. 2017 T100797.

\section{References}

[1] Pullen J M. Enabling Military Coalition Command and Control with Interoperating Simulations as a System of Systems[C] 5th International Conference on Simulation and Modeling Methodologies, Technologies and Applications. 2016:409-417.

[2] Pullen J M. Enabling Military Coalition Command and Control with Interoperating Simulations as a System of Systems[M] Simulation and Modeling Methodologies, Technologies and Applications. Springer International Publishing, 2016.

[3] Hiebert J, Gallimore R, Stigler J W. A Knowledge Base for the Teaching Profession: What Would It Look like and How Can We Get One?[J]. Educational Researcher, 2002, 31(5):3-15.

[4] Rudnik K. System with probabilistic fuzzy knowledge base and parametric inference operators in risk assessment of innovative projects[M]. Pergamon Press, Inc. 2015.

[5] Galinec D, Steingartner W. A Look at Observe, Orient, Decide and Act Feedback Loop, Pattern-Based Strategy and Network Enabled Capability for Organizations Adapting to Change[J]. Acta Electrotechnica Et Informatica, 2013, 13.

[6] Joiner H V. Threat assessment orchestrator system and method: US, US6742128[P]. 2004. 